\title{
In vitro evaluation of shear bond strength of resin cements to computer-aided design/computer-aided manufacturing (CAD/CAM) framework materials
}

\author{
Guler Yildirim Avcu \\ Department of Prosthodontics, Faculty of Dentistry, Inonu University, Malatya, Turkey \\ E-mail: guler_yldrm@hotmail.com
}

\begin{abstract}
The aim of this in vitro study was to compare the shear bond strength (SBS) of three different resin cements to different computer-aided design/computer-aided manufacturing (CAD/CAM) framework materials (cobalt-chromium, titanium, and zirconia). Specimens $(12 \times 2 \mathrm{~mm})$ were divided into three different main groups according to materials $(n=10)$; Cr-Co discs (Hard metal; HM), titanium discs $(\mathrm{T})$, and zirconia discs $(\mathrm{Z})$ and embedded in acrylic resin, and smoothed with silicon carbide papers and sandblasting with $50 \mu \mathrm{m}$ aluminum oxide. Specimens of each framework materials were divided into three subgroups; (1) Universal resin cement, (2) Syringeable resin ionomer cement, and (3) Self-adeziv resin cement. The shear bond strength test was conducted using a universal testing machine. Data (MPa) was analyzed using one way ANOVA, Kruskal Wallis tests, and post-hoc tests. There was no statistically significant difference between SBS value of universal resin cement and self-adhesive cement for each material $(\mathrm{P}>0.05)$. Resin ionomer cement showed the lowest SBS value $(\mathrm{P}<0.05)$. No significant differences in SBS were found between framework materials. The SBS values of self-adhesive cements are as high as universal resin cements for hard metal, titanium and zirconium supported restorations.
\end{abstract}

Keywords: Shear bond strength, hard metal milling, titanium, zirconia.

DOI: $10.7176 / \mathrm{JSTR} / 5-2-11$

\section{Introduction}

With the development of technology, there have been changes in the production methods of the frameworks used in the construction of prosthetic restorations in dentistry. While casting technique has been used in metal frameworks production for many years, new restorative treatment options and production methods are used with the production of CAD/CAM and laser sinter devices (Alghazzawi, 2016, Barazanchi et al., 2017). Nowadays, fixed and removable denture frameworks have produced milling of hard metal, soft metal, titanium, and zirconia with CAD/CAM, and laser sintering (LS) besides the lost wax technique (Kocaagaoglu et al., 2017, Kocaagaoglu et al., 2016). Hard alloy milling that metal frameworks have been prepared by milling nonporous hard alloy blocks is a subtractive production method (Onoral et al., 2018). Compared to hard-metal milling, soft metal milling decreases stress on the milling machine, increases the life span of milling bur and reduces milling time for production of metalceramic restorations (Kocaagaoglu et al., 2017, Kocaagaoglu et al., 2016). The process periods in the manufacture of metal alloy blocks are similar to those in the manufacture of zirconia blocks (Kocaagaoglu et al., 2016).

Titanium that has been used in dental implants for many years has increased its use in metal ceramic restorations, cast restorations or partial dentures due to its biocompatibility and optimum mechanical properties (Almilhatti et al., 2013). However, low bond strengths between titanium and composite veneering systems have been reported. Several methods that are based on macra- and micromechanical retention, chemical bonding, or a combination of both mechanicisms, have been used to increase the bonding of resin composite to titanium (Almilhatti et al., 2013). Pure titanium and titanium alloys have

93 | P a g e

www.iiste.org 
biocompatible, low allergenic potential, high corrosion resistance and low cost (Antanasova et al., 2018, Bankoglu Gungor and Karakoca Nemli, 2018). The disadvantages of titanium are the high melting temperature, poor castability and required application of special manufacture processes/equipment. To overcome these limitations, $\mathrm{CAD} / \mathrm{CAM}$ has begun to be used for the fabrication of titanium prosthetic frameworks. Nevertheless, titanium-porcelain connections have been reported to be poor, resulting in failures of porcelain veneer. Therefore titanium ceramic bonding is tried to be improved and ultra-lowfusing porcelains with firing temperatures below $800{ }^{\circ} \mathrm{C}$ have been used for veneering (Antanasova et al., 2018).

Good retention has been assumed to be one of the major necessities in accomplishing long-term success of restorations; therefore researchers have attempted to increase retention studies. A variety of different cements are used to lute restorations and recently, an excessive number of resin cements have been introduced on the dental market. Adhesive systems can be classified according to the application stages as etch\&rinse (total-etch) adhesive systems (Rosa et al., 2015), self-etch adhesive systems (Manso and Carvalho, 2017) and resin modified glass ionomer adhesives (Francois et al., 2018)

The null hypotheses to be tested were: 1- the type of the framework materials and 2- the type of resin cements does not affect the shear bond strength of cements to material surfaces.

\section{Material Methods}

The three different main groups of framework materials $(12 \times 2 \mathrm{~mm})$ were prepared from Cr-Co (Hard metal; HM, Kera-disc, Eisenbacher), titanium (T, Kera Ti-5 disc, Eisenbacher), and zirconia (Z, ceramaill, Amann Girrbach) ( $n=10)$. CAD/CAM milling (Ceramill, Amann Girrbach), sinter furnace (Ceramill Argotherm, Amann Girrbach) and (D15, Yenadent) were used in the preparation of specimens that were embedded in acrylic resin (Palapress Vario, Heraeus Kulzer) and smoothed with silicon carbide papers (600 and 800 grits) and sandblasting with $50 \mu \mathrm{m}$ aluminum oxide. Specimens of each framework material were divided into three subgroups, which received one of the following luting cement: (1) Self adeziv resin cement (Supercem, DentKist) (2) Syringeable resin ionomer cement (Infinity, DenMat) (3) Universal resin cement (Panavia V5, Kuraray).

Custom-made teflon molds (an internal diameter of $3 \mathrm{~mm}$ and thickness of $3 \mathrm{~mm}$ ) were placed on the surface of the specimens. Self-adhesive resin cement and syringeable resin ionomer cement based on direct application resin. After application of cements light-cure the surface of specimen for according to the manufacturer's recommendation and a LED (Woodpecker Med. Instrument) was used for light polymerization for 20 seconds. Before using the universal resin cement, specimens were applied primer, and blowed dry with air. After application of cement, the surface of specimen was light-cured for 10 seconds based on the manufacturer's recommendation. The specimens were stored in $37^{\circ} \mathrm{C}$ distilled water for 24 hour before being tested for SBS. Test specimens were fixed in a universal testing machine (MTS Criterion ${ }^{\circledR}$ Series 42). Shear loading was applied at the interface of the cement and the framework material at $0.5 \mathrm{~mm} / \mathrm{min}$ crosshead speed. The maximum debonding force was recorded for each specimen.

\subsection{Statistical Analysis}

Analyses were performed with statistical software (IBM SPSS Statistics v23.0, IBM Corp, Chicago, USA). The normality of the data distribution was evaluated by the Shapiro-Wilk test. Data (MPa) wasanalyzed using one way ANOVA and Kruskal Wallis tests to perform a comparison between each two groups used Tukey HDS and Bonferroni correction Man Whitney U tests. $(p<0.05)$

\section{Results}

The means and medians of SBS values (MPa), standard deviation, and range of the different cements and framework materials are presented in Table 1. There were significant differences between the types of cements in all groups $(\mathrm{P}<0.05)$. There was no significant difference among materials at the same cement. The lowest SBS values $(10.5,11.7$, and 9.2 MPa) were found syringeable resin ionomer cement groups $(\mathrm{P}<0.05)$. There was no significant difference between SBS values of self-adeziv resin cement and universal resin cement in all groups $(\mathrm{P}>0.05)$. 
Table 1. Values of shear bond strength (standard deviations and minimum-maximum) of the test groups.

\begin{tabular}{|l|l|l|l|l|}
\hline Materials & \multicolumn{2}{|l|}{ Cements } & \multicolumn{2}{l|}{} \\
& Panavia & Infinity & Supercem & $P$ \\
\hline $\mathrm{HM}$ & $13.2( \pm 2.1)^{\mathrm{a}}$ & $10.5( \pm 10.2)^{\mathrm{b}}$ & $11.8( \pm 2.6)^{\mathrm{a}}$ & $0.001^{\mathrm{a}}$ \\
\hline $\mathrm{T}$ & $14.4(11.7-16.1)^{\mathrm{a}}$ & $11.7(9.9-12.8)^{\mathrm{b}}$ & $14.5(12.2-15.3)^{\mathrm{a}}$ & $0.032^{*} *$ \\
\hline $\mathrm{Z}$ & $12.9(9.9-16.6)^{\mathrm{a}}$ & $9.2(7.2-10.6)^{\mathrm{b}}$ & $13.5(11.3-15.5)^{\mathrm{a}}$ & $0.007 * *$ \\
\hline$P^{* *}$ & 0.801 & 0.94 & 0.376 & \\
\hline
\end{tabular}

HM; Hard Metal, T; Titanium, Z; Zirconium, values followed by different lowercase letters in the same row show statistical differences. *One way anova and tukey HDS, **Kruskall Wallis and Benforroni correction Man Whitney U tests.

\section{Discussion}

The bonding of resin cements to the framework materials is an important factor in the success of the final prosthetic restorations. Various factors may affect the strength of such a bond, especially the type of luting cements and the framework materials used. This study indicated that results of SBS are significantly different among the tested cements, and thus the null hypothesis 2 was rejected.

Limited reports have been published on the bonding of resin cements to hard metal and titanium. In the present study, hard metal, titanium, and zirconia framework materials was compared. When the same cement system was used, no significant difference was found between the bond strengths of framework materials. But there was a significant difference between bond strengths of cements. Different bonding mechanisms may occur due to the variation of chemical components. In addition, a variety of surface treatments such as chemical and/or electrical etching (Krueger et al., 1990), airborne particle abrasion (Okada et al., 2019), used metal primer and silica coating (Dal Piva et al., 2019) have been documented (Hattar et al., 2014) and are supposedly used to enhance the bond strength of the restoration to the cement. Sandblasting the metallic alloys with aluminum oxide is commonly employed for surface cleaning and a proper retentive topography, with a consequent increase in the adhesive bond (Fonseca et al., 2012).

In the present study, Panavia V5 and Supercem yielded significantly higher bond strength values than Infinity, possibly because the bond performance of this cement lies in its chemical composition. In the literature, studies of Infinity and Supercem cements are limited; there is also no study regarding bonding with these framework materials investigated with the two types of cement. Few studies evaluating the bond strength between Panavia V5 and CAD/CAM resin blocks and monolithic zirconium materials are found in the literature.(Higashi et al., 2016, Wen et al., 2017) However, there is no study evaluating the bond strength between Panavia V5 and chromium-cobalt and titanium. Musani et al. compared the micro tensile bond strength of two metal bonding resin cements to sandblasted cobalt chromium alloy. In contrast to this study, the bond strength of self-adhesive cement was found to be higher (Musani et al., 2013).

Larabi et al. investigated bonding strength of zirconia on dentin using different adhesive systems. According to the results of the study, there was no significant difference between the bond strength of resin modified glass ionomer cement (9.69 Mpa) and Panavia V5 (12.94 Mpa) and some of the selfadhesive cements (14.86 Mpa). However, there is a difference between bond strength of Panavia V5 and some of the self-adhesive cements (4.76-5.11 Mpa). In the present study, Panavia V5 showed the SBS value (12.9 Mpa).

The limitation of this study is that no thermal cycles were made, different surface treatments were not applied and no more cement was used.

\section{Conclusions}

Universal resin cements require more steps in the application phase; tooth isolation breaks down due to saliva and lack of time for cementation. Self-adhesive cements can be applied in one step. According to the results of this study, the bond strength of self-adhesive cements is as high as universal cements.

95 | P a g e

www.iiste.org 


\section{References}

Alghazzawi, T. F. (2016). Advancements in CAD/CAM technology: Options for practical implementation. Journal of Prosthodontic Research 60, 72-84.

Almilhatti, H. J., Neppelenbroek, K. H., Vergani, C. E., Machado, A. L., Pavarina, A. C. \& Giampaolo, E. T. (2013). Adhesive bonding of resin composite to various titanium surfaces using different metal conditioners and a surface modification system. Journal of Applied Oral Science, 21, 590-596.

Antanasova, M., Kocjan, A., Kovac, J., Zuzek, B. \& Jevnikar, P. (2018). Influence of thermomechanical cycling on porcelain bonding to cobalt-chromium and titanium dental alloys fabricated by casting, milling, and selective laser melting. Journal of Prosthodontic Research, 62, 184-194.

Bankoglu Gungor, M. \& Karakoca Nemli, S. (2018). The Effect of Resin Cement Type and Thermomechanical Aging on the Retentive Strength of Custom Zirconia Abutments Bonded to Titanium Inserts. The International Journal of Oral \& Maxillofacial Implants, 33, 523-529.

Barazanchi, A., Li, K. C., Al-Amleh, B., Lyons, K. \& Waddell, J. N. (2017). Additive Technology: Update on Current Materials and Applications in Dentistry. Journal of Prosthodontics, 26, 156163.

Da Rosa, W. L. D. O., Piva, E. \& Da Silva, A. F. (2015). Bond strength of universal adhesives: A systematic review and meta-analysis. Journal of Dentistry, 43, 765-776.

Dal Piva, A. M., Carvalho, R. L., Lima, A. L., Bottino, M. A., Melo, R. M. \& Valandro, L. F. (2019). Silica coating followed by heat-treatment of MDP-primer for resin bond stability to yttriastabilized zirconia polycrystals. Journal of Biomedical Materials Research Part B: Applied Biomaterials, 107, 104-111.

Fonseca, R. G., Martins, S. B., De Oliveira Abi-Rached, F. \& Dos Santos Cruz, C. A. (2012). Effect of different airborne-particle abrasion/bonding agent combinations on the bond strength of a resin cement to a base metal alloy. Journal of Prosthetic Dentistry, 108, 316-23.

Francois, P., Vennat, E., Le Goff, S., Ruscassier, N., Attal, J.-P. \& Dursun, E. (2018). Shear bond strength and interface analysis between a resin composite and a recent high-viscous glass ionomer cement bonded with various adhesive systems. Clinical Oral Investigations, 1-10.

Hattar, S., Hatamleh, M., Khraisat, A. \& Al-Rabab'ah, M. (2014). Shear bond strength of selfadhesive resin cements to base metal alloy. Journal of Prosthetic Dentistry, 111, 411-5.

Higashi, M., Matsumoto, M., Kawaguchi, A., Miura, J., Minamino, T., Kabetani, T., Takeshige, F., Mine, A. \& Yatani, H. (2016). Bonding effectiveness of self-adhesive and conventional-type adhesive resin cements to $\mathrm{CAD} / \mathrm{CAM}$ resin blocks. Part 1: Effects of sandblasting and silanization. Dental Materials Journal, 35, 21-28.

Kocaagaoglu, H., Albayrak, H., Kilinc, H. I. \& Gumus, H. O. (2017). Effect of repeated ceramic firings on the marginal and internal adaptation of metal-ceramic restorations fabricated with different CAD-CAM technologies. Journal of Prosthetic Dentistry, 118, 672-677. 
Kocaagaoglu, H., Kilinc, H. I., Albayrak, H. \& Kara, M. (2016). In vitro evaluation of marginal, axial, and occlusal discrepancies in metal ceramic restorations produced with new technologies. Journal of Prosthetic Dentistry, 116, 368-74.

Krueger, G. E., Diaz-Arnold, A., Aquilino, S. \& Scandrett, F. (1990). A comparison of electrolytic and chemical etch systems on the resin-to-metal tensile bond strength. Journal of Prosthetic Dentistry, 64, 610-617.

Manso, A. P. \& Carvalho, R. M. 2017. Dental Cements for Luting and Bonding Restorations: SelfAdhesive Resin Cements. Dental Clinics of North America, 61, 821-834.

Musani, S., Musani, I., Dugal, R., Habbu, N., Madanshetty, P. \& Virani, D. (2013). An in vitro Comparative Evaluation of Micro Tensile Bond Strength of Two metal bonding Resin Cements bonded to Cobalt Chromium alloy. Journal of International Oral Health, 5, 73-8.

Okada, M., Taketa, H., Torii, Y., Irie, M. \& Matsumoto, T.(2019). Optimal sandblasting conditions for conventional-type yttria-stabilized tetragonal zirconia polycrystals. Dental Materials, 35, 169175.

Onoral, O., Ulusoy, M., Seker, E. \& Etikan, I. (2018). Influence of repeated firings on marginal, axial, axio-occlusal, and occlusal fit of metal-ceramic restorations fabricated with different techniques. Journal of Prosthetic Dentistry, 120, 415-420.

Wen, M., Bebsh, M., Kaaber, M., Salomon, C. \& França, R. (2017). Shear bond strength of lithium disilicate using various adhesive cement. Dental Materials, 33, e11. 